\title{
Avaliação dos tipos de glaucoma no serviço de oftalmologia da UNICAMP
}

\author{
Evaluation of glaucoma types at the sector of ophthalmology-UNICAMP
}

\author{
Andréia Peltier Urbano ${ }^{1}$ \\ Telma Gondim Freitas ${ }^{2}$ \\ Enyr Saram Arcieri ${ }^{2}$ \\ Alessandra Peltier Urbano ${ }^{3}$ \\ Vital Paulino Costa ${ }^{4}$
}

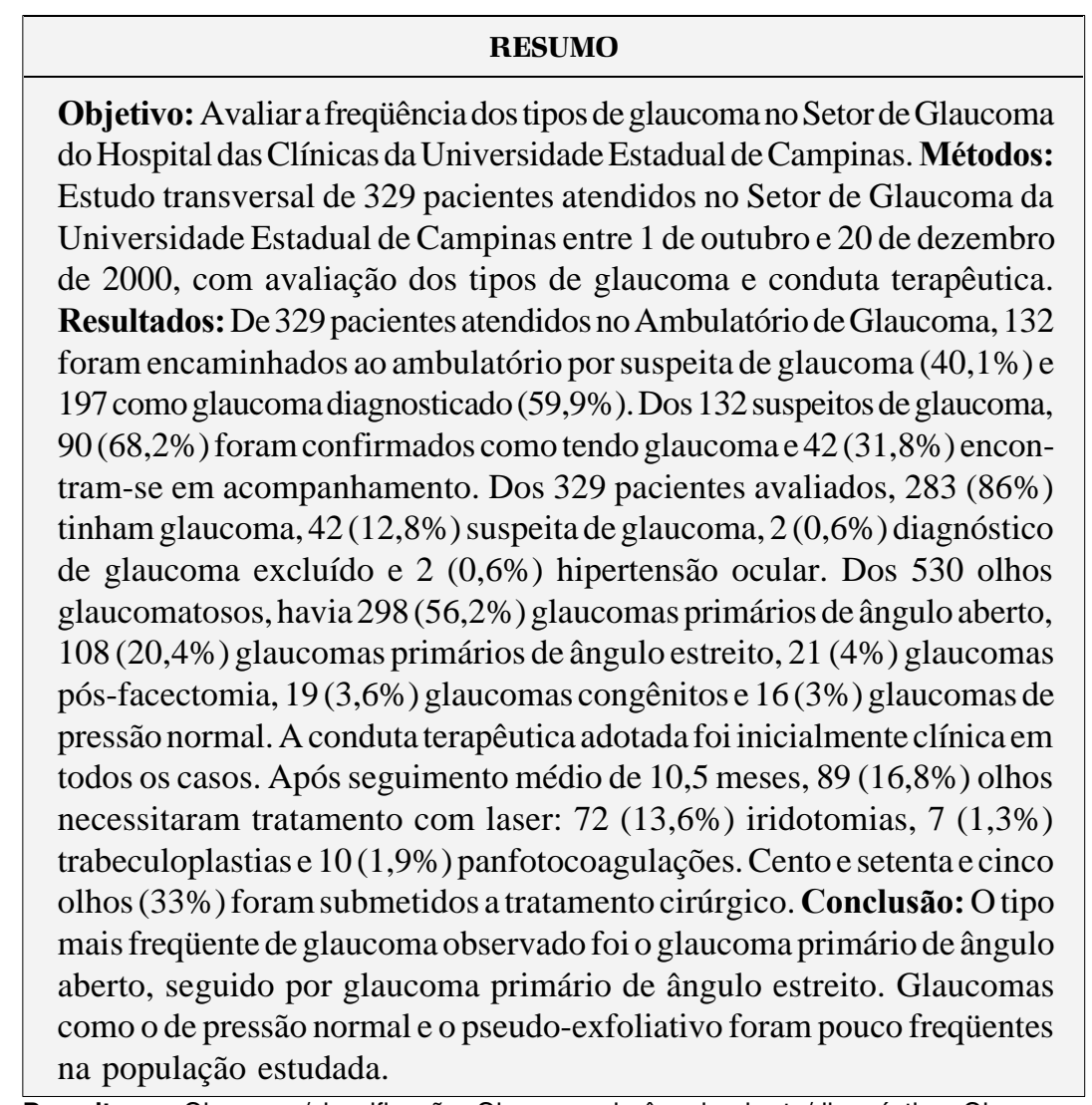

Descritores: Glaucoma/classificação; Glaucoma de ângulo aberto/diagnóstico; Glaucoma de ângulo fechado/diagnóstico; Glaucoma de ângulo aberto/terapia; Glaucoma de ângulo fechado/terapia; Glaucoma de ângulo aberto/cirurgia; Glaucoma de ângulo fechado/cirurgia; Trabeculectomia; Estudos transversais.

Médica Residente de Oftalmologia da Universidade Estadual de Campinas - UNICAMP.

${ }^{2}$ Médico(a) Estagiário(a) do Setor de Glaucoma do Departamento de Oftalmologia da UNICAMP

3 Acadêmica de Medicina da Universidade Federal da Bahia - UFBA.

${ }^{4}$ Chefe do Setor de Glaucoma do Departamento de Oftalmologia da UNICAMP, Professor Livre Docente pela Faculdade de Medicina da USP

Endereço para correspondência: Rua Napoleão de Barros, 1058 Ap.22 - São Paulo (SP) CEP 04024-003. E-mail: andreiaurbano@uol.com.br

Recebido para publicação em 13.07.2001 Aceito para publicação em 22.07.2002

Nota Editorial: Pela análise deste trabalho e por sua anuência na divulgação desta nota, agradecemos ao Dr. Carlos Rubens L. Figueiredo.

\section{INTRODUÇ̃̃̃O}

INTRODUÇÃOO

A estimativa do número de indivíduos com glaucoma em todo o mundo para o ano 2000 foi de 66,8 milhões de pessoas, com cerca de 8 milhões apresentando cegueira bilateral devido à lesão irreversível do nervo óptico $^{(1)}$. As diferentes formas de glaucoma constituem a principal causa de cegueira irreversível nos países industrializados e em desenvolvimento ${ }^{(2)}$.

O glaucoma é uma neuropatia óptica de causa multifatorial, caracterizada pela lesão progressiva do nervo óptico, com conseqüente repercussão no campo visual. Apesar de poder cursar com pressões intra-oculares consideradas dentro dos padrões da normalidade (glaucoma de pressão normal), a elevação da pressão intra-ocular é seu principal fator de risco.
\end{abstract}


Trata-se de doença assintomática que requer uma investigação pormenorizada com exames do nervo óptico (NO), pressão intra-ocular (PIO) e campo visual para o seu diagnóstico ${ }^{(3)}$. Estima-se que a metade dos casos de glaucoma permanece não diagnosticada ${ }^{(2)}$.

O presente estudo objetiva a avaliação dos tipos mais freqüentes de glaucoma no ambiente universitário e da conduta terapêutica adotada, pretendendo acrescentar dados inéditos para a literatura nacional a respeito da proporção dos diferentes tipos de glaucoma presentes numa amostra da população brasileira.

\section{MÉTODOS}

Realizou-se um estudo de corte transversal, com a avaliação de 329 pacientes atendidos no Ambulatório de Glaucoma do Setor de Oftalmologia do Hospital das Clínicas da Universidade Estadual de Campinas no período de $1^{\circ}$ de outubro a 20 de dezembro de 2000.

No questionário aplicado foram investigados: sexo, idade, data do $1^{\circ}$ atendimento na UNICAMP (realizado no Setor de Oftalmologia Geral), motivo do encaminhamento ao ambulatório de glaucoma (suspeita de glaucoma ou glaucoma diagnosticado), tipo de glaucoma definido no ambulatório, uni ou bilateralidade e conduta adotada (clínica ou cirúrgica).

Definiu-se como glaucoma primário a presença de alteração do nervo óptico (NO) e alteração do campo visual típicas de glaucoma. A alteração do NO foi caracterizada pela presença de pelo menos dois dos seguintes critérios: escavação do nervo óptico $>0,7$, perda de rima localizada, hemorragia de disco ou assimetria da escavação do nervo óptico $>0,3$. Definiu-se defeito de campo visual glaucomatoso de acordo com um dos seguintes critérios: 2 ou mais pontos contíguos com perda maior ou igual a 10 decibéis (db) nas áreas de Bjerrum superior ou inferior, comparadas para controles de mesma idade ou 3 ou + pontos contíguos, com perda maior ou igual a $5 \mathrm{db}$ nas áreas de Bjerrum superior ou inferior ${ }^{(4)}$. A definição de glaucoma secundário incluiu aumento de pressão intra-ocular de causa estabelecida, sem necessidade de lesão de nervo óptico ou de campo visual para diagnóstico. Os glaucomas primários foram subdivididos em:

- glaucoma primário de ângulo aberto (GPAA): PIO $\geq 21$ mmHg sem medicação hipotensora ocular, ângulo aberto, lesão do NO e defeito de CV, idade > 40 anos

- glaucoma primário de ângulo estreito (GPAE): PIO $\geq 21$ $\mathrm{mmHg}$, ângulo estreito, lesão do NO, defeito de CV

- glaucoma de pressão normal (GPN): PIO < $21 \mathrm{mmHg}(6$ medidas diárias, das 8 às 18 horas), com demais critérios semelhantes ao GPAA

- glaucoma juvenil: idade $>10$ e $<40$ anos, com demais critérios semelhantes ao GPAA

- glaucoma congênito: $\mathrm{PIO} \geq 21 \mathrm{mmHg}$ sem medicação, diâmetro corneano aumentado, idade de aparecimento $<4$ anos
Definiu-se como hipertensão ocular PIO $\geq 21 \mathrm{mmHg}$ sem alteração do $\mathrm{NO}$ e sem defeito de CV.

A análise dos dados foi realizada no programa SPSS for MS Windows, versão 9.0. As variáveis contínuas foram descritas pela média e desvio padrão, enquanto as variáveis categóricas foram descritas quanto às proporções.

\section{RESULTADOS}

Foram avaliados 329 pacientes atendidos no Ambulatório de Glaucoma durante o período de $1^{\circ}$ de outubro a 20 de dezembro de 2000.

A amostra dos pacientes avaliados era composta por 155 indivíduos do sexo feminino $(54,8 \%)$ e 128 pacientes do sexo masculino (45,2\%). A média de idade foi $60,92 \pm 17,49$ anos.

Dos 329 pacientes avaliados, 132 foram encaminhados ao ambulatório por suspeita de glaucoma (40,1\%) e 197 como glaucoma diagnosticado $(59,9 \%)$. Dos 132 suspeitos de glaucoma, $90(68,2 \%)$ foram confirmados como tendo glaucoma e $42(31,8 \%)$ encontram-se em acompanhamento.

Assim, dos 329 pacientes, 86\% $(n=283)$ apresentavam glaucoma, $12,8 \%(n=42)$ eram suspeitos, $0,6 \%(n=2)$ apresentavam hipertensão ocular e $0,6 \%(\mathrm{n}=2)$ não tinham glaucoma.

Dos 283 pacientes com glaucoma, 87,3\% $(n=247)$ apresentavam glaucoma bilateral e $12,7 \%(\mathrm{n}=36)$ unilateral, resultando num total de 530 olhos glaucomatosos. Destes, 449 (84,7\%) foram classificados como glaucoma primário e $81(15,3 \%)$ glaucoma secundário.

A Tabela 1 revela os tipos de glaucoma observados neste estudo. Os mais freqüentes foram: glaucoma primário de ângulo aberto (GPAA) - $(56,2 \%, n=298)$, glaucoma primário de ângulo estreito (GPAE) - $(20,4 \%, \mathrm{n}=108)$, glaucoma pós-facectomia $(4 \%, n=21)$, glaucoma congênito $-(3,6 \%, n=19)$ e glaucoma de pressão normal $(\mathrm{GPN})$ - $(3 \%, \mathrm{n}=16)$. Dentre os glaucomas bilaterais, os mais freqüentes foram: GPAA $(60,3 \%$; $n=298)$, GPAE $(21,9 \% ; n=108)$, glaucoma congênito $(3,7 \%, n=9)$ e glaucoma de pressão normal $(3,2 \% ; n=8)$. Dos 36 glaucomas unilaterais, os mais freqüentes foram: pós-facectomia $(36,1 \%$, $\mathrm{n}=13)$, traumático $(19,4 \%, \mathrm{n}=7)$, neovascular $(13,8 \%, \mathrm{n}=5)$ e inflamatório $(8,3 \%, \mathrm{n}=3)$.

Todos os pacientes receberam inicialmente orientação para começar tratamento medicamentoso. A análise retrospectiva da evolução destes pacientes evidenciou que 33,03\% $(n=175)$ dos casos necessitaram de intervenção cirúrgica, enquanto $50,18 \%(n=266)$ dos casos mantiveram-se recebendo exclusivamente terapêutica medicamentosa. Os 89 olhos restantes $(16,79 \%)$ foram submetidos ao tratamento a laser, seguido ou não de tratamento medicamentoso. Dos casos estudados, 13,01\% $(\mathrm{n}=69)$ foram submetidos à trabeculectomia (TREC) simples, e 12,45\% $(\mathrm{n}=66)$ a TREC com antimetabólito (mitomicina), 7,16\% $(\mathrm{n}=38)$ a cirurgia combinada de trabeculectomia e facectomia com lente intra-ocular (LIO) e $0,18 \%$ $(n=1)$ a cirurgia combinada sem LIO. Trabeculoplastia foi realizada em $1,32 \%(n=7)$, iridotomia em $13,58 \%(n=72)$ e panfoto- 


\begin{tabular}{|c|c|c|c|c|c|c|}
\hline \multicolumn{7}{|c|}{ Tabela 1. Tipos de glaucoma mais freqüentes } \\
\hline & \multicolumn{2}{|c|}{ Geral } & \multicolumn{2}{|c|}{ Bilateral } & \multicolumn{2}{|c|}{ Unilateral } \\
\hline & $\mathbf{N}$ & $\%$ & $\mathbf{N}$ & $\%$ & $\mathbf{N}$ & $\%$ \\
\hline \multicolumn{7}{|l|}{ GLAUCOMAS PRIMÁRIOS } \\
\hline Glaucoma primário de ângulo aberto & 298 & 56,2 & 298 & 56,2 & 0 & 0 \\
\hline Glaucoma primário de ângulo estreito & 108 & 20,4 & 108 & 20,4 & 0 & 0 \\
\hline Congênito & 19 & 3,6 & 18 & 3,4 & 1 & 0,2 \\
\hline Glaucoma pressão normal & 16 & 3,0 & 16 & 3,0 & 0 & 0 \\
\hline Juvenil & 8 & 1,5 & 8 & 1,5 & 0 & 0 \\
\hline \multicolumn{7}{|l|}{ GLAUCOMAS SECUNDÁRIOS } \\
\hline \multirow{2}{*}{$\begin{array}{ll}\text { Pós-facectomia } & \text { Com lente intra-ocular } \\
\text { Sem lente intra-ocular }\end{array}$} & 13 & 2,5 & 4 & 0,8 & 9 & 1,7 \\
\hline & 8 & 1,5 & 4 & 0,8 & 4 & 0,8 \\
\hline Anomalia/Síndrome-Axenfeld Rieger & 10 & 1,9 & 10 & 1,9 & 0 & 0 \\
\hline Inflamatório & 9 & 1,7 & 6 & 1,1 & 3 & 0,6 \\
\hline Traumático & 7 & 1,3 & 0 & 0 & 7 & 1,3 \\
\hline Pseudo-exfoliativo & 7 & 1,3 & 6 & 1,1 & 1 & 0,2 \\
\hline Pigmentar & 6 & 1,1 & 6 & 1,1 & 0 & 0 \\
\hline Neovascular & 5 & 0,9 & 0 & 0 & 5 & 0,9 \\
\hline Facomórfico & 3 & 0,6 & 2 & 0,4 & 1 & 0,2 \\
\hline Facolítico & 2 & 0,4 & 2 & 0,4 & 0 & 0 \\
\hline Crise glaucomatociclítica & 2 & 0,4 & 2 & 0,4 & 0 & 0 \\
\hline Cortisônico & 2 & 0,4 & 2 & 0,4 & 0 & 0 \\
\hline Outras anomalias do desenvolvimento ocular & 2 & 0,4 & 2 & 0,4 & 0 & 0 \\
\hline Pós-ceratoplastia penetrante & 2 & 0,4 & 0 & 0 & 2 & 0,4 \\
\hline Ciclite heterocrômica & 1 & 0,2 & 0 & 0,2 & 1 & 0 \\
\hline Glaucoma maligno & 1 & 0,2 & 0 & 0,2 & 1 & 0 \\
\hline Sturge-Weber & 1 & 0,2 & 0 & 0 & 1 & 0,2 \\
\hline Total de olhos & 530 & & 494 & & 36 & \\
\hline
\end{tabular}

coagulação em 1,88\% $(\mathrm{n}=10)$ dos olhos. Onze casos $(2,07 \%)$ necessitaram ser submetidos ao implante de tubo, $1(0,18 \%)$ à ciclocrioterapia e $1(0,18 \%)$ à pancrioterapia (Tabela 2$)$.

\section{DISCUSSÃO}

Evidenciou-se que $40,1 \%$ dos pacientes encaminhados ao ambulatório o são por suspeita de glaucoma e que, destes, $31,1 \%$ não tiveram o diagnóstico definido durante o período do

\begin{tabular}{|c|c|c|c|c|}
\hline \multicolumn{5}{|c|}{ Tabela 2. Conduta terapêutica } \\
\hline & & & N (Olhos) & $\%$ \\
\hline \multicolumn{3}{|l|}{ Apenas Clínica } & 266 & 50,18 \\
\hline \multicolumn{3}{|l|}{ Laser } & 89 & 16,79 \\
\hline \multicolumn{3}{|l|}{ Iridotomia } & 72 & 13,58 \\
\hline \multicolumn{3}{|c|}{ Trabeculoplastia } & 7 & 1,32 \\
\hline \multicolumn{3}{|c|}{ Panfotocoagulação } & 10 & 1,88 \\
\hline \multicolumn{3}{|c|}{ Cirurgia } & 175 & 33,03 \\
\hline \multirow{2}{*}{\multicolumn{2}{|c|}{ Trabeculectomia }} & Simples & 69 & 13,01 \\
\hline & & Mitomicina & 66 & 12,45 \\
\hline \multirow{2}{*}{ Combinada } & Con & lente intra-ocular & 38 & 7,17 \\
\hline & Sen & lente intra-ocular & 1 & 0,18 \\
\hline \multicolumn{3}{|l|}{ Implantes } & 11 & 2,07 \\
\hline \multicolumn{3}{|c|}{ Ciclocrioterapia } & 1 & 0,18 \\
\hline \multicolumn{3}{|c|}{ Pancrioterapia } & 1 & 0,18 \\
\hline
\end{tabular}

estudo, necessitando de maior avaliação para confirmação ou exclusão diagnóstica. Vuori e Nikoskelainen ${ }^{(3)}$, investigando pacientes com suspeita de glaucoma encaminhados a um hospital universitário na Finlândia, relataram que, de 175 pacientes referidos, em 54 casos (31\%) o diagnóstico de glaucoma não pôde ser confirmado, taxa concordante com a deste estudo.

Dos 283 pacientes com glaucoma diagnosticado nesta amostra, $87,3 \%$ eram bilaterais e $12,7 \%$ unilaterais, $84,7 \%$ primários e $15,3 \%$ secundários. Teikari e O'Donnell ${ }^{(5)}$, analisando dados de registro hospitalar na Finlândia, descreveram que de 48.864 pacientes adultos glaucomatosos examinados, $67 \%$ correspondiam a glaucomas primários e 33\% a glaucomas secundários.

A avaliação diagnóstica da amostra estudada evidenciou que apenas $0,6 \%(n=2)$ dos casos apresentaram hipertensão ocular, contrastando com estudos populacionais, que demonstram maior prevalência de hipertensão ocular do que glau$\operatorname{coma}^{(6)}$. A baixa freqüência de hipertensão ocular encontrada pode ser explicada pelo fato do estudo ter sido realizado no ambiente hospitalar, com encaminhamento ao ambulatório especializado de glaucoma, durante período limitado de estudo de aproximadamente dois meses. É possível que, por se tratar de ambiente com nível terciário de atendimento, casos com pior prognóstico visual sejam artificialmente selecionados, reduzindo a frequiência de pacientes com hipertensão ocular.

A avaliação dos tipos mais freqüentes de glaucoma encon- 
trados na população brasileira não tem sido objeto de estudos. Apesar do presente relato analisar pacientes seguidos em Serviço Universitário e não uma amostra representativa da população brasileira, é possível obter informações que, ainda que parcialmente, indiquem quais as formas de glaucoma mais freqüentes no nosso meio.

É importante ressaltar que a freqüência dos tipos de glaucoma varia de acordo com a população estudada. A maior freqüência dos glaucomas primários (GPAA e GPAE) encontrada na amostra é concordante aos relatos de alguns autores. Bartosova e $\operatorname{cols}^{(7)}$, avaliando pacientes com diferentes tipos de glaucoma tratados numa clínica de olhos em Praga, descreveram 54,1\% (n=74) de GPAA, 24,8\% ( $\mathrm{n}=34)$ de GPAE, 9,5\% ( $\mathrm{n}=13)$ de glaucoma congênito, $8,8 \%(\mathrm{n}=12)$ de glaucoma pigmentar e $2,9 \%(n=4)$ de glaucoma exfoliativo. Pode-se observar que a frequiência de GPAA e GPAE é similar ao estudo descrito, porém encontraram-se taxas menores dos outros tipos de glaucoma.

Na maioria das populações, o GPAE é menos comum do que o GPAA, porém há inversão desta frequiência no Canadá, Alaska e Groelândia ${ }^{(8)}$. Congdon et al. ${ }^{(9)}$ relatou que a prevalência de GPAE é 20 a 40 vezes maior em esquimós do que em brancos e 10 a 15 vezes maior em chineses que em brancos. Shiose et al. ${ }^{(10)}$, em estudo populacional de 8.126 habitantes de 7 regiões do Japão, evidenciaram que o GPAE é mais freqüente em japoneses do que caucasianos. Em contrapartida, relatos provenientes da África demonstraram baixa freqüência de GPAE. Kaimbo et al. ${ }^{(11)}$, analisando 176 indivíduos glaucomatosos numa comunidade urbana no Congo, observaram $72 \%$ de GPAA, 9\% de glaucoma do afácico, 6\% de glaucoma inflamatório, $3 \%$ de glaucoma pseudoexfoliativo, $2,2 \%$ de glaucoma traumático, 1,07\% de glaucoma secundário a catarata, $1,7 \%$ de GPN, $1,7 \%$ de glaucoma congênito, $1,3 \%$ de glaucoma neovascular, $0,6 \%$ de glaucoma pigmentar, $0,6 \%$ de glaucoma cortisônico, 0,6 \% de glaucoma associado a retinoblastoma, e nenhum caso de GPAE. No presente estudo, o GPAE foi o segundo tipo de glaucoma mais encontrado, dado concordante com o padrão de frequiência da maioria dos estudos ${ }^{(5)}$.

O GPN é relatado com maior freqüência em determinadas populações, principalmente em japoneses. No estudo populacional realizado por Shiose et al. ${ }^{(10)}$ avaliando 8.126 indivíduos, GPN foi diagnosticado em 2,04\% dos casos, hipertensão ocular em 1,37\%, enquanto apenas $0,58 \%$ tiveram GPAA, $0,34 \%$ GPAE e $0,60 \%$ outros tipos de glaucoma. Os autores enfatizam que a alta prevalência de GPN no Japão pode refletir uma peculiaridade social na manifestação da pressão intraocular $^{(8)}$. Dos 167 pacientes glaucomatosos examinados por Bechmann et al. ${ }^{(12)}$ num hospital universitário na Alemanha, $32,06 \%$ apresentaram GPN, 18,32\% glaucoma pseudoexfoliativo, 30,53\% GPAE, $15,26 \%$ GPAA, 9,92\% glaucoma pigmentar e 16,79\% hipertensão ocular. Ao contrário destes estudos, o presente relato demonstra que a freqüência de GPN em um Serviço Universitário no Brasil é de cerca de 3\%. Devemos ressaltar que a confirmação da ausência de picos pressóricos maiores que $21 \mathrm{mmHg}$ foi realizada por meio de curvas tensionais de duração de 10 horas. Assim, é possível que parte dos pacientes com GPN detectados neste estudo apresentem, na realidade, GPAA com pico pressórico não medido, e que, portanto, a frequiência de GPN no nosso meio seja ainda menor. Por outro lado, é possível que a detecção de GPN por oftalmologistas gerais seja deficiente, o que artificialmente diminuiria o número de encaminhamentos a um setor especializado.

Outros tipos de glaucoma estão descritos em maior freqüência em determinadas áreas. O glaucoma pigmentar é bem descrito em Caucasianos ${ }^{(12)}$, enquanto o glaucoma pseudoexfoliativo é mais encontrado em Escandinavos e em pacientes de origem Mediterrânea ${ }^{(13)}$. Ringvold et al. ${ }^{(14)}$, no estudo de três municípios da Noruega, descreveram prevalência de glaucoma pseudoexfoliativo variando entre $10-21 \%$ em indivíduos com idade superior a 64 anos. Neste estudo, pôde-se observar que as freqüências dos glaucomas pigmentar e pseudo-exfoliativo são inferiores às encontradas nos demais relatos, provavelmente devido à composição étnica da população brasileira.

A avaliação da terapêutica dos casos estudados demonstrou que a conduta cirúrgica foi realizada em $33 \%$ dos casos, iridotomia a laser em $13,58 \%$, concordante com o relato de Bartosova et al.(7), que realizaram $26,5 \%$ de cirurgias para o glaucoma e $15,7 \%$ iridotomias em pacientes com diferentes tipos de glaucoma tratados numa clínica de olhos em Praga. A freqüência de trabeculoplastia (1,32\%) no presente estudo, no entanto, foi inferior ao estudo de Bartosova $(18,8 \%)^{(7)}$. A baixa freqüência dos glaucomas pigmentar e pseudoexfoliativo tipos de glaucoma que apresentam boa resposta a trabeculoplastia, e alta prevalência de glaucomas avançados nesta amostra contribuíram para a indicação limitada de trabeculoplastias em nosso Serviço.

\section{CONCLUSÃO}

Neste estudo foram evidenciados os tipos de glaucoma seguidos no Setor de Glaucoma da UNICAMP. Concluímos que o glaucoma primário de ângulo aberto e o glaucoma primário de ângulo estreito foram os mais encontrados. Ao contrário de outras populações, o glaucoma de pressão normal e o glaucoma pseudoexfoliativo foram pouco freqüentes na população deste estudo.

\section{ABSTRACT}

Purpose: To evaluate the frequency of different types of glaucoma at the Hospital das Clínicas of Campinas State University. Methods: Cross-sectional study of 329 patients followed at the Glaucoma Service of Campinas State University from October 1 to December 20, 2000. The frequency of each type of glaucoma and the treatment were analyzed. Results: There were 329 patients examined at the Glaucoma Service: $132(40.1 \%)$ were referred to the service as glaucoma 
suspects and 197 (59.9\%) as glaucoma patients. Ninety of the 132 glaucoma suspects had glaucoma (68.2\%) and 42 are still under investigation (31.8\%). Among the 329 patients, 283 $(86 \%)$ had glaucoma, $42(12.8 \%)$ were glaucoma suspects, 2 $(0.6 \%)$ had ocular hypertension and $2(0.6 \%)$ did not have glaucoma. There were 530 eyes with glaucoma: 298 (56.2\%) with primary open angle glaucoma, 108 (20.4\%) with chronic angle closure glaucoma, 21 (4\%) with glaucoma following cataract surgery, $19(3.6 \%)$ congenital glaucoma and $16(3 \%)$ with low-tension glaucoma. All patients received initial clinical treatment with IOP-lowering medication. After a mean follow up of 10.5 months, 89 (16.8\%) underwent laser therapy: $72(13.6 \%)$ iridotomy, $7(1.3 \%)$ trabeculoplasty and $10(1.9 \%)$ panphotocoagulation. A hundred and seventy nine (33\%) eyes required surgical treatment. Conclusion: The most frequent types of glaucoma were primary open angle and angle closure glaucoma. Low tension glaucoma and glaucoma associated with exfoliation syndrome were uncommon in this population.

Keywords: Glaucoma/classification; Open angle glaucoma/diagnosis; Angle closure glaucoma/diagnosis; Open angle glaucoma/therapy; Angle closure glaucoma/therapy; Open angle glaucoma/surgery; Angle closure glaucoma/surgery; Trabeculectomy; Cross sectional studies.

\section{REFERÊNCIAS}

1. Coleman AL. Glaucoma. Lancet 1999;354:1803-10.

2. Krieglstein GK. Blindness caused by glaucoma. Ophthalmologe 1993;90:554-6.

3. Vuori ML, Nikoskelainen E. Evaluation of glaucoma patients referred to a university clinic during one year. Acta Ophthalmol Scand 1997;75:692-4.

4. Costa, VP. Perimetria computadorizada: um guia básico de interpretação. Rio de Janeiro: RioMed; 1995

5. Teikari JM, O'Donnell J. Epidemiologic data on adult glaucomas. Data from the Hospital Discharge Registry and the Registry of Right to Free Medication. Acta Ophthalmol (Copenh) 1989;67:184-91.

6. Shields MB. Open-Angle Glaucomas. In: Shields MB. Textbook of glaucoma. $5^{\text {th }}$ ed. Baltimore:Williams and Wilkins; 1998;153-76.

7. Bartosova L, Kraus H, Ruzickova E. Working ability of patients with various forms and stages of glaucoma. Cesk Oftalmol 1989;45:127-37.

8. Shields MB. Pupillary-block glaucomas. In: MB. Textbook of glaucomas. $5^{\text {th }}$ ed Baltimore: Williams and Wilkins;1998;177-94.

9. Congdon NG, Youlin Q, Quigley H, Hung PT, Wang TH, Ho TC, et al. Biometry and primary angle-closure glaucoma among Chinese, white, and black populations. Ophthalmology 1997;104:1489-95.

10. Shiose Y, Kitazawa Y, Tsukahara S, Akamatsu T, Mizokami K, Futa R, et al. Epidemiology of glaucoma in Japan-a nationwide glaucoma survey. Jpn J Ophthalmol 1991;35:133-55.

11. Kaimbo WA, Kaimbo D, Missotten L. Glaucoma in Congo. Bull Soc Belge Ophtalmol 1997;267:21-6.

12. Bechmann M, Thiel MJ, Roesen B, Ullrich S, Ulbig MW, Ludwig K. Central corneal thickness determined with optical coherence tomography in various types of glaucoma. Br J Ophthalmol 2000;84:1233-7.

13. Shields MB. Glaucomas associated with disorder of the lens. In: Shields MB. Textbook of glaucoma. $5^{\text {th }}$ ed. Baltimore: Williams and Wilkins; 1998; 252-68

14. Ringvold A, Blika S, Elsas T, Guldahl J, Brevik T, Hesstvedt P, et al. The prevalence of pseudo-exfoliation in three separate municipalities of MiddleNorway. A preliminary report. Acta Ophthalmol 1987;182(Suppl):17-20. 